\title{
Systematic review and meta-analysis on the efficacy of methotrexate in rheumatoid arthritis
}

\author{
Xiaofan Jiang ${ }^{1}$, Jin Zeng ${ }^{2}$, Fang Chen ${ }^{3}$, Juane $\mathrm{Li}^{1}$ \\ ${ }^{1}$ Department of Chinese Medicine, Shaanxi Provincial People's Hospital, Xi'an, China; ${ }^{2}$ Anorectal Department, Xi'an Hospital of Traditional Chinese \\ Medicine, Xi'an, China; ${ }^{3}$ Shaanxi Institute of Food and Drug Control, Xi'an, China \\ Contributions: (I) Conception and design: X Jiang, J Li; (II) Administrative support: J Li; (III) Provision of study materials or patients: X Jiang, J Zeng, \\ F Chen; (IV) Collection and assembly of data: All authors; (V) Data analysis and interpretation: X Jiang, J Zeng, J Li; (VI) Manuscript writing: All \\ authors; (VII) Final approval of manuscript: All authors. \\ Correspondence to: Juane Li. 256 Youyi Xi Lu, Xi’an, China. Email: lizhuan-1980@126.com.
}

\begin{abstract}
Backgroundk Rheumatoid arthritis (RA) is a chronic autoimmune disease dominated by chronic inflammation of the synovium of the joints. Methotrexate (MTX) is the most widely used in the treatment of RA. This study systematically evaluated the clinical efficacy of MTX on RA and provided a theoretical basis for the clinical treatment of RA.

Methods: Four English databases (PubMed, Embase, Medline, and Web of Sciences) were searched for randomized controlled trials (RCTs) on MTX treatment of RA published from the date of establishment of the database to 2021. The Cochrane Handbook 5.0.2 was used to perform risk bias evaluation and Review Manager 5.3 was used to conduct a meta-analysis.

Results: A total of eight articles were included. The meta-analysis showed that, compared to the control group, the number of patients with DAS28-ESR $\leq 2.6$ [erythrocyte sedimentation rate (ESR)] in the MTX alone or MTX combined treatment groups was higher, and the incidence of adverse events was also higher. However, there was no significant difference in the level of alanine aminotransferase (ALT) after treatment versus the control group.

Discussion: MTX alone or MTX combined treatment can better control the condition of RA patients without causing damage to the patient's blood system or liver and kidney function, but may increase the probability of adverse events.
\end{abstract}

Keywords: Methotrexate (MTX); rheumatoid arthritis (RA); clinical efficacy; meta-analysis

Submitted Aug 08, 2021. Accepted for publication Sep 27, 2021.

doi: 10.21037/apm-21-2471

View this article at: https://dx.doi.org/10.21037/apm-21-2471

\section{Introduction}

Rheumatoid arthritis (RA) is a chronic autoimmune system disease characterized by inflammatory synovitis. Clinically, it manifests as chronic, symmetrical, and progressive multiple joint pain or progressive destruction. In severe cases, it even causes the erosion and destruction of cartilage, subchondral bone, and tendons, and eventually leads to joint deformities or loss of mobility $(1,2)$. RA is a global disease, with an incidence rate of between $0.5 \%$ and $1 \%$ worldwide. In China, the incidence of RA is approximately $0.42 \%$. It is more common in women, especially those aged 50-75 years old. In late stage of RA, patients will develop joint deformities, and their quality of life will be significantly affected (3).

The pathogenesis of RA is still unclear. Irreversible bone destruction occurs in the early stage (4), and it currently cannot be cured radically. Treatment mainly involves alternative and symptomatic treatment, which aim to relieve the clinical symptoms of patients and reduce joint injury (5), and includes psychotherapy, drug therapy, and surgical treatment. Drug therapy can reduce 
joint pain and inflammation (6); commonly used drugs include non-steroidal anti-inflammatory drugs (NSAIDs) and disease modifying anti-rheumatic drugs (DMARDs), glucocorticoids (GCs), and biologic agents (7). Of the four types of drugs, NSAIDs primarily inhibit the production of prostaglandins to achieve anti-inflammatory and analgesic effects, thereby reducing joint pain (8). Although DMARDs do not have anti-inflammatory effects, they can reduce synovial inflammation as well as bone and joint destruction in patients to delay the disease (9). GCs have strong antiinflammatory and analgesic effects and can quickly improve joint pain in RA patients, but adverse reactions, such as osteoporosis and aseptic osteonecrosis, will increase after long-term use (10). Biologic agents can intervene in immune links, such as $\mathrm{T}$ and $\mathrm{B}$ cell activation, to exert anti-inflammatory effects; they are effective but expensive, and their long-term efficacy is not clear (11). It remains undetermined which drug is the first choice for treatment of RA. The best treatment plan is formulated according to the patient's individual conditions, while avoiding adverse reactions.

Methotrexate (MTX) is a typical DMARD. It is an effective drug with a reasonable price and acceptable side effects $(12,13)$, and is thus widely used in the treatment of RA. Taken orally, MTX can be basically eliminated within 24 hours. After entering the cell, part of the MTX produces metabolite polyglutamate MTX (MTXPG) under the action of polyglutamate synthetase, and MTXPG plays a decisive role in the treatment of RA (14). However, there are still $30-40 \%$ of patients receiving MTX treatment that are not achieving the ideal therapeutic effects and are prone to tolerance (15). Thus, it is inferred that MTX combined with other drugs may be an effective way to improve its clinical efficacy in RA. Generally, a single anti-rheumatic drug is not effective, or patients with progressive, poor prognosis, and refractory rheumatoid arthritis can be treated with a combination of anti-rheumatic drugs with different mechanisms. When combined medication, its adverse reactions are not necessarily more than single medication. Therefore, the choice of therapeutic drugs needs to be combined with the actual situation of the patient.

So far, there have been a large number of clinical controlled trials to analyze the clinical efficacy of MTX combination therapy for RA, but there are many types of combination drugs, and there is no systematic review of the treatment of RA. In addition, the quality of the literature is uneven, and it is difficult to obtain a unified clinical trial. Based on the above reasons, in order to make a more complete evaluation of the clinical efficacy and safety of MTX in the treatment of RA, and to provide a theoretical basis for the rational clinical application of drugs in the treatment of RA, this study conducted a meta-analysis of the clinical trials of MTX in the treatment of RA, so as to systematically evaluate the clinical efficacy and safety of MTX in the treatment of RA, and provide evidence-based evidence for the clinical drug treatment of RA. We present the following article in accordance with the PRISMA reporting checklist (available at https://dx.doi.org/10.21037/ apm-21-2471).

\section{Methods}

\section{Inclusion and exclusion criteria}

The literature was identified according to the following inclusion criteria: (I) the subjects were diagnosed with RA according to the RA classification criteria established by the American Association of Rheumatology (ACR), and were aged $\geq 18$ years, with no limitation on the gender; (II) RCTs on MTX in the treatment of RA published in English; and (III) the experimental group was treated by MTX alone or MTX combined with other drugs, and the baseline data of the two groups was comparable.

The exclusion criteria were as follows: (I) the research subjects were non-RA patients, animals, children, or cells; (II) the experimental group was not treated by MTX alone or MTX combined with other drugs; (III) unpublished degree theses or non-English literature; (IV) RCTs involving RA patients accompanied by other diseases; (V) low quality literature with obvious bias; and (VI) RCTs with incomplete data or repeated publications.

\section{Literature retrieval}

Four English databases (PubMed, Embase, Medline, and Web of Sciences) were searched for RCTs on the treatment of RA using MTX from the date of establishment of the database to 2021. The search terms were "rheumatoid arthritis", "methotrexate", "Clinical trial", "DMARD", "RA", and "MTX", and they were connected by "OR" or "AND".

\section{Literature screening}

Initially, some duplicates were eliminated. A second screening was then performed after reading the titles and abstracts. Next, according to the inclusion criteria and 
exclusion criteria, a third screening was conducted.

\section{Data extraction}

Two experts used unified Microsoft Excel (https://www. microsoft.com/zh-cn/microsoft-365/excel) to independently collate the data. Inconsistencies was resolved by discussion or arbitration by a third expert. The following data were collated: (I) research title, first author's name, publication year, and publication name; (II) general information of the research object: region, sample size, gender ratio, and age distribution; (III) observation indicators; and (IV) intervention methods in the control and experimental groups.

\section{Risk bias evaluation}

The risk of bias assessment included seven items: random sequence, allocation concealment, blinding method for the subjects, blinding method for the outcome assessor, research data, selective reports, and other biases.

\section{Statistical analysis}

The risk bias assessment tool provided by Cochrane Handbook for Systematic Reviews of Interventions 5.0.2 (https://training.cochrane.org/handbook/archive/v5.0.2/) was used to perform risk bias assessment. The $\mathrm{I}^{2}$ test was used to evaluate the heterogeneity; If $\mathrm{I}^{2} \geq 50 \%$, the random effects model (REM) was used, whereas if $\mathrm{I}^{2}<50 \%$, the fixed effects model (FEM) was used. The combined effect size was assessed using the $U$ test and $95 \%$ confidence interval (CI), and the result of the $\mathrm{U}$ test was expressed by $\mathrm{P}$ value. A $\mathrm{P}$ value $<0.05$ was the threshold for significance.

\section{Results}

\section{Literature retrieval results}

Initially, a total of 826 articles were identified, of which 319 were retrieved from PubMed, 152 were retrieved from Embase, 144 were retrieved from Medline, and 211 were retrieved from Web of Sciences. Next, NoteExpress 3.2 software (http://www.inoteexpress.com/aegean/) was used to eliminate duplicates. Of the 641 remaining articles, 555 were eliminated after reading the titles and abstracts. Of the remaining 86 articles, 78 were excluded, as they did not meet the inclusion and exclusion criteria. Finally, a total of eight articles were included in this study (Figure 1).

\section{Bias risk assessment results}

As shown in Table 1, all of the eight included articles reported the use of random grouping methods. Among them, seven RCTs $(16,18-23)$ described specific random methods, suggesting low risk, whereas the remaining RCT (17) did not mention the specific random method, suggesting an unclear risk. Also, three of the eight articles $(16,21,22)$ clearly mentioned that the grouping was doubleblind, indicating a low risk, while the other five $(17-20,23)$ did not mention whether the blinding method was used, suggesting unclear risk. Furthermore, four of the eight articles $(17,20,21,23)$ clearly mentioned that the patients signed the informed consent, indicating a high risk, while the other four $(16,18,19,22)$ did not mention whether the subjects were blinded, indicating an unclear risk. The outcome data of all the eight articles (16-23) was complete, indicating a low risk. Also, all eight articles (16-23) were not selective reports and did not have other biases, suggesting a low risk. Detailed results are shown in Figures 2,3.

\section{$D A S 28-E S R \leq 2.6$}

Six of the eight included articles reported on the DAS28ESR $\leq 2.6$ after treatment of RA using MTX (16,19-23). The difference in the number of remissions after treatment between the experimental group and the control group was compared. The heterogeneity test found no significant heterogeneity among the six included studies $\left(\mathrm{I}^{2}=0 \%\right.$, $\mathrm{P}=0.54$ ), so the FEM was used for analysis (Figure 4). The combined effect size of meta-analysis was mean difference $(\mathrm{MD})=1.66 ; 95 \% \mathrm{CI}: 1.40-1.97 ; \mathrm{Z}=5.78 ; \mathrm{P}<0.00001$, which suggested significant differences in the number of remissions between the two groups. Figure 5 displays the funnel chart of DAS28-ESR $\leq 2.6$. The results showed that the scattered points in the funnel chart fell within the $95 \%$ CI, indicating little publication bias.

\section{Alanine aminotransferase (ALT)}

Five of the eight included articles reported on ALT after MTX treatment of RA $(17,18,20-22)$. The difference in ALT levels after treatment between the experimental group and the control group was compared. It was found that the five studies were quite heterogeneous $\left(\mathrm{I}^{2}=90 \%, \mathrm{P}<0.00001\right)$, so the REM was used (Figure 6). The combined effect size 


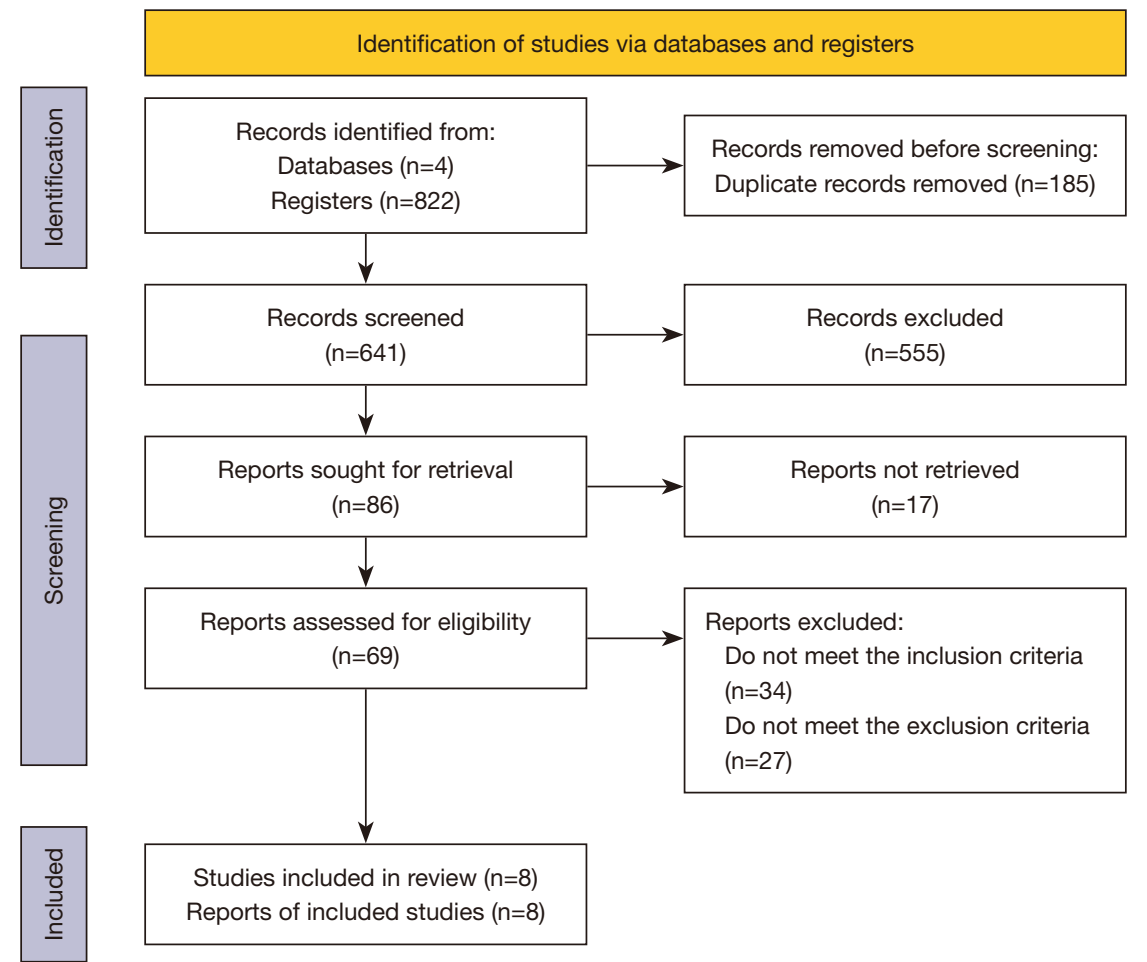

Figure 1 Literature retrieval process.

Table 1 Basic information of the included literature

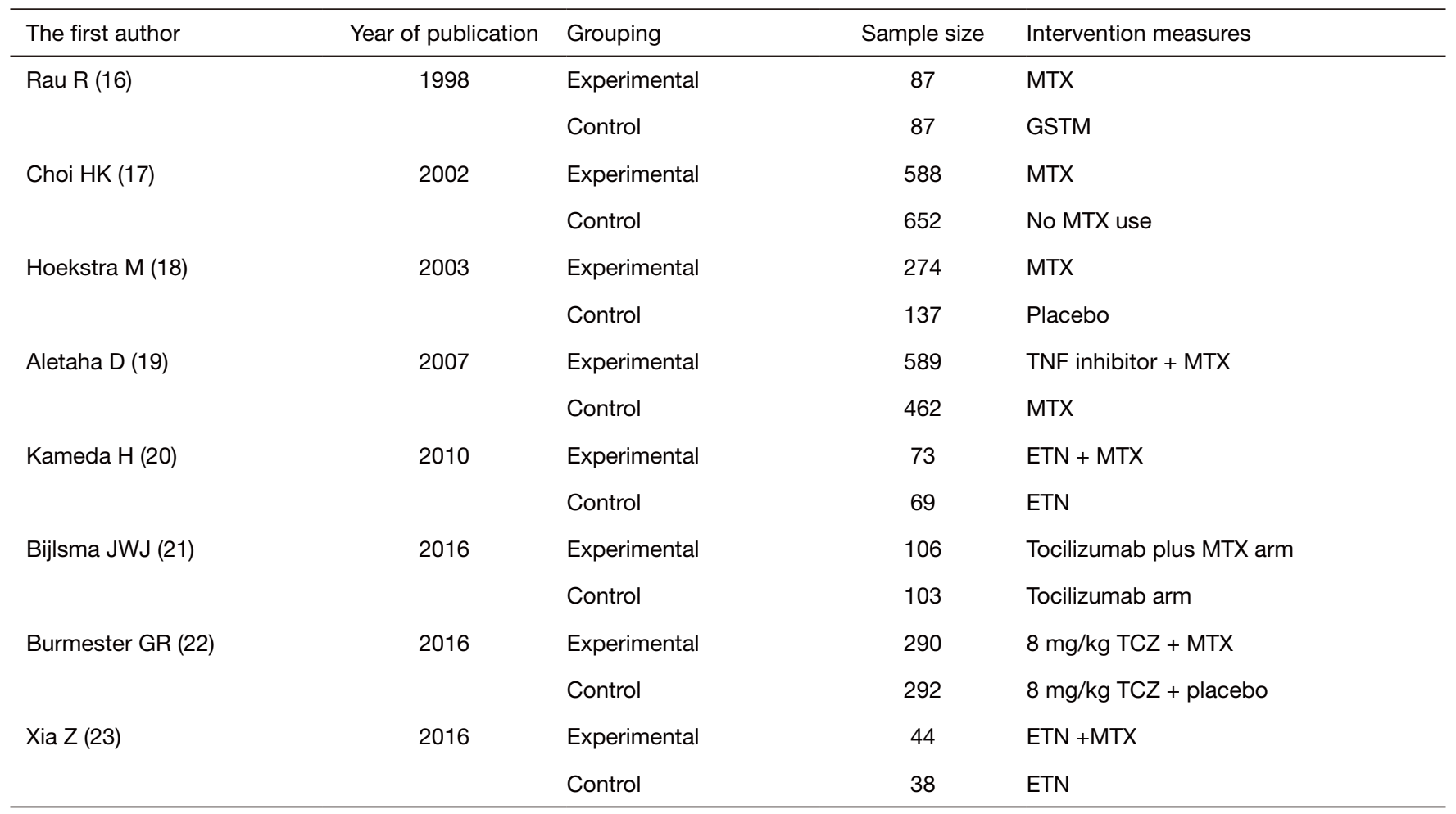

MTX, methotrexate; ETN, etanercept; TCZ, tocilizumab. 


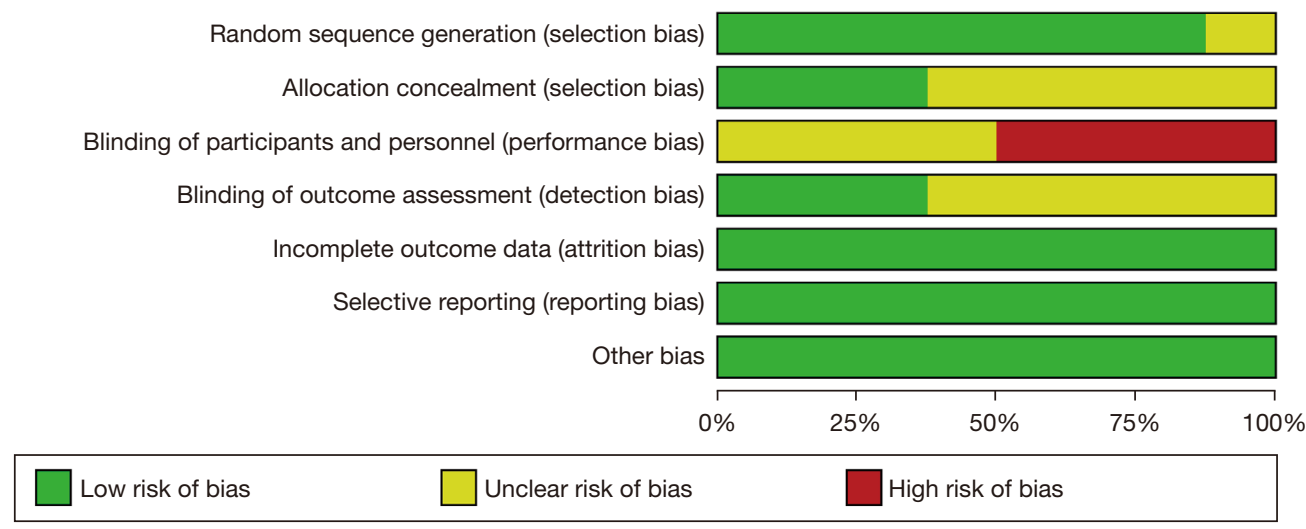

Figure 2 Bias risk bar chart.

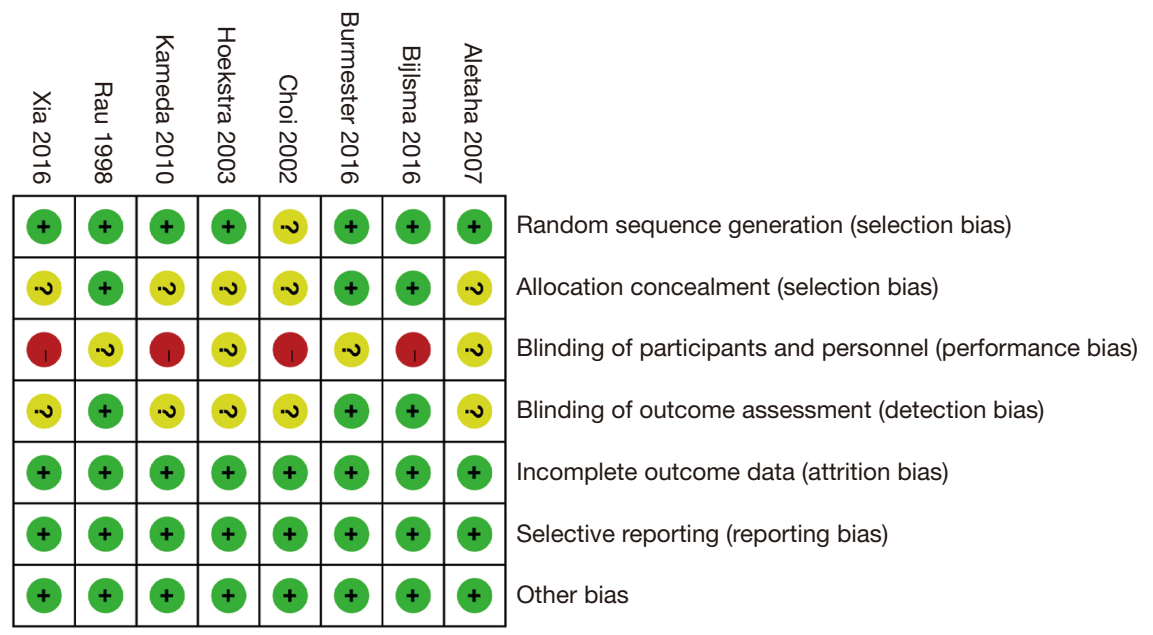

Figure 3 Bias risk.

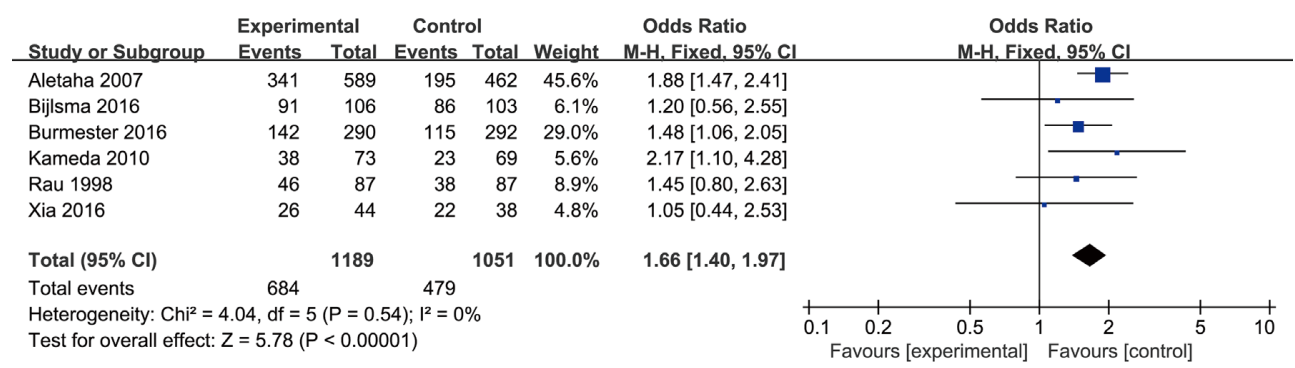

Figure 4 Forest plot of DAS28-ESR $\leq 2.6$. ESR, erythrocyte sedimentation rate.

of meta-analysis was $\mathrm{MD}=0.66 ; 95 \%$ CI: $0.28-1.52 ; \mathrm{Z}=0.99$; $\mathrm{P}=0.32$, which suggested that there was no significant difference in the ALT levels between the two groups. Figure
7 displays a funnel chart of ALT. The results showed that some of the scattered points in the funnel chart fell outside the $95 \% \mathrm{CI}$, and the distribution was scattered, indicating a 


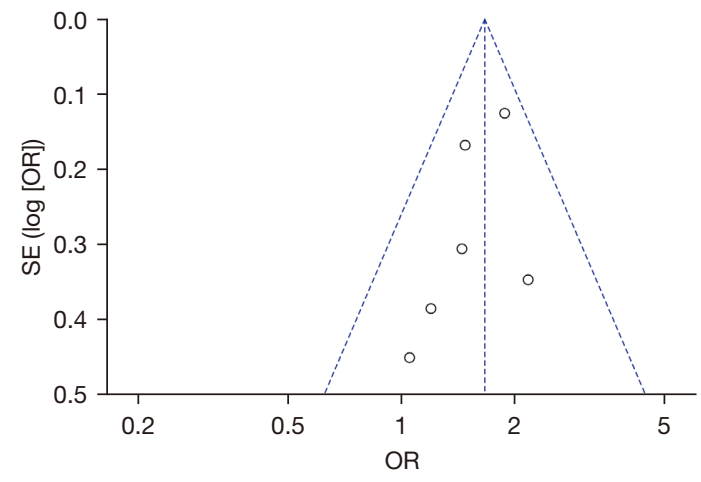

Figure 5 Funnel chart showing DAS28-ESR $\leq 2.6$. ESR, erythrocyte sedimentation rate.

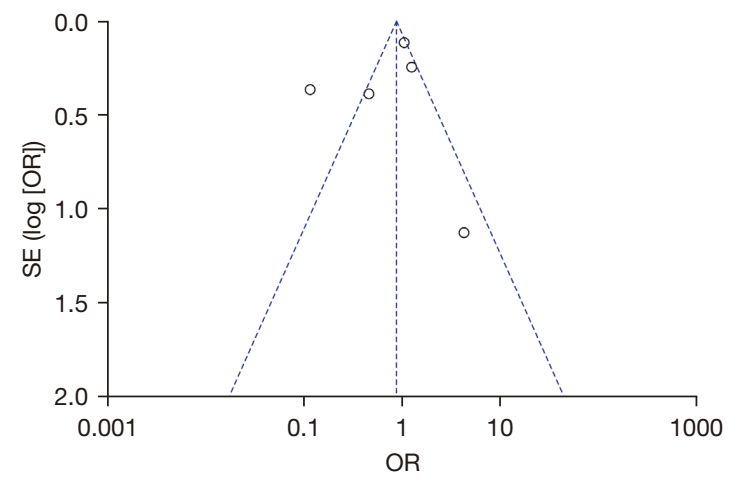

Figure 7 Funnel chart showing ALT. ALT, alanine aminotransferase.

\begin{tabular}{|c|c|c|c|c|c|c|}
\hline \multirow[b]{2}{*}{ Study or Subgroup } & \multicolumn{2}{|c|}{ Experimental } & \multicolumn{2}{|c|}{ Control } & \multirow{2}{*}{\multicolumn{2}{|c|}{ Weight $M-H_{1}$}} \\
\hline & Events & Total & Events & Total & & \\
\hline Bijlsma 2016 & 105 & 106 & 99 & 103 & $9.2 \%$ & \\
\hline Burmester 2016 & 256 & 290 & 250 & 292 & $23.4 \%$ & \\
\hline Choi 2002 & 311 & 588 & 336 & 652 & $24.9 \%$ & \\
\hline Hoekstra 2003 & 11 & 274 & 36 & 137 & $21.4 \%$ & \\
\hline Kameda 2010 & 47 & 73 & 55 & 69 & $21.0 \%$ & \\
\hline Total $(95 \% \mathrm{Cl})$ & & 1331 & & 1253 & $100.0 \%$ & \\
\hline Total events & 730 & & 776 & & & \\
\hline
\end{tabular}

Odds Ratio

H. Random. 95\% C $4.24[0.47,38.61]$

$1.26[0.78,2.05]$

$1.06[0.84,1.32]$

$0.12[0.06,0.24]$

$0.46[0.22,0.98]$

$0.66[0.28,1.52]$

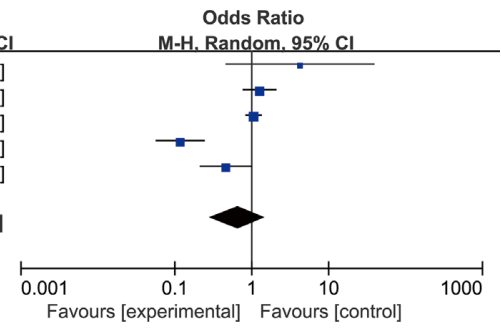

Figure 6 Forest plot showing ALT. ALT, alanine aminotransferase.

certain degree of publication bias.

\section{The incidence of adverse events}

Four of the eight included reported on the incidence of adverse events after MTX treatment of RA (16,21-23). The difference in the incidence of adverse events between the experimental group and the control group after receiving treatment was compared. The heterogeneity test found no significant heterogeneity among the four studies $\left(\mathrm{I}^{2}=0 \%\right.$, $\mathrm{P}=0.55$ ), so the FEM was used (Figure 8 ). The combined effect size of meta-analysis was $M D=1.46 ; 95 \% \mathrm{CI}$, 1.10, 1.92; $\mathrm{Z}=2.65 ; \mathrm{P}=0.008$, which suggested significant differences in the incidence of adverse events between the two groups. Figure 9 displays a funnel chart of the incidence of adverse events. The results showed that the scattered points in the funnel chart all fell within the $95 \%$ CI, suggesting little publication bias.

\section{Discussion}

RA is a chronic autoimmune disease, which manifests pathologically as chronic inflammation of joint synovium, formation of pannus, joint bone destruction, deformity, and loss of function (24). RA tends to occur in the proximal fingertip joints, wrist joints, elbow joints, toe joints, etc. Clinically, it manifests as symmetrical, persistent joint swelling and tenderness around the diseased joints. In the later stages, RA will cause severe damage to joint function, and even lead to physical disability of the patient $(25,26)$. Since RA causes irreversible bone destruction, treatment is aimed at inhibiting or reducing this irreversible bone destruction and protecting the patient's joint mobility to the greatest extent. RA cannot be radically cured (27), so prevention of bone and joint damage is critical to avoid the loss of joint function in patients. MTX is a DMARD that is widely used in the treatment of RA, and its clinical efficacy has been recognized by numerous clinical studies. It is currently the first choice for the treatment of RA (28). Studies have found that for some mild patients, one DMARD can achieve ideal effects, but in most cases, several DMARDs are usually used in combination to effectively control the disease. At present, a large number of clinical trials have confirmed that the clinical effect of combined treatment is better $(29,30)$. 


\begin{tabular}{|c|c|c|c|c|c|}
\hline \multirow[b]{2}{*}{ Study or Subgroup } & \multicolumn{2}{|c|}{ Experimental } & \multicolumn{2}{|c|}{ Control } & \multirow[b]{2}{*}{ Weight } \\
\hline & Events & Total & Events & Total & \\
\hline Bijlsma 2016 & 14 & 106 & 5 & 103 & $5.3 \%$ \\
\hline Burmester 2016 & 136 & 290 & 115 & 292 & $73.1 \%$ \\
\hline Rau 1998 & 28 & 87 & 21 & 87 & $17.1 \%$ \\
\hline Xia 2016 & 5 & 44 & 4 & 38 & $4.6 \%$ \\
\hline Total $(95 \% \mathrm{Cl})$ & & 527 & & 520 & $100.0 \%$ \\
\hline Total events & 183 & & 145 & & \\
\hline \multicolumn{6}{|c|}{ Heterogeneity: $\mathrm{Chi}^{2}=2.10, \mathrm{df}=3(\mathrm{P}=0.55) ; \mathrm{I}^{2}=0 \%$} \\
\hline Test for overall effect & 2.65 & 0.008 & & & \\
\hline
\end{tabular}

Figure 8 Forest plot showing the incidence of adverse events.

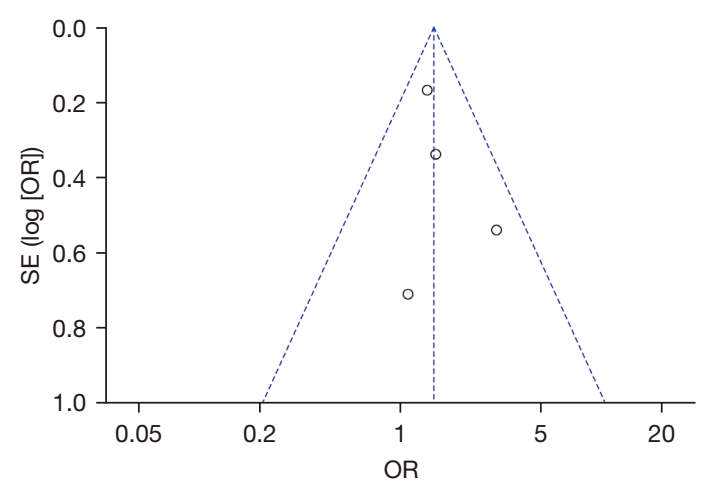

Figure 9 Funnel chart showing the incidence of adverse events.

Biological agents are a kind of biological products that can target the downstream inflammatory factors of rheumatoid arthritis (31). Common drugs include tofacitinib. Biological drugs have the advantages of quick onset and good curative effect (32). However, the effects of hepatitis, tuberculosis, and tumor diseases need to be excluded before administration. At the same time, the cost of the drug is relatively high, so it is difficult to be widely used in clinical practice.

Because rheumatoid arthritis is an autoimmune disease with a high disability rate and can cause long-term chronic inflammation in the patient's body, after combined drug treatment, it may cause more serious adverse reactions, so the patient's prognosis is often poor. At the same time, the cost of rheumatoid arthritis treatment drugs is relatively high, which brings a huge economic burden to the patient's family and society. Therefore, it is recommended to improve the clinical diagnosis rate of the disease, optimize the patient's awareness of the disease, strictly standardize the diagnosis and treatment process of the disease, select effective treatment drugs, and strengthen patient care, thus improving the diagnosis and treatment of rheumatoid arthritis. In this study, meta-analysis was conducted on RCTs of the clinical treatment of RA using MTX alone or
MTX combined with other drugs. A total of eight articles were included, involving 3,891 subjects. The two groups were compared for DAS28-ESR, ALT levels, and the incidence of adverse events. The DAS28 scoring system is a comprehensive and clinically recognized evaluation method. Erythrocyte sedimentation rate (ESR) is an important inflammatory index for clinicians to judge the disease condition of RA patients. DAS28-ESR $\leq 2.6$ means symptom remission. The results of this study showed that MTX alone or MTX combined with other drugs had a higher number of symptom remissions after treatment versus the control group; that is, MTX alone or MTX combined with other drugs could better control the condition of RA patients. ALT reflects the damage to the blood system, as well as the liver and kidney function, of patients with RA. Elevated ALT indicates damage to liver cells. This study found that MTX alone or MTX combined treatment did not increase the patients' ALT levels. Finally, the incidence of adverse events was compared, and it was found that the MTX alone or MTX combined treatment groups were more likely to have adverse events compared to the control group, which suggested that the two treatments may have the same mechanism of action and pharmacokinetics.

\section{Conclusions}

This study was a systematic review of RCTs on the clinical efficacy of MTX in the treatment of RA. It was found that MTX alone or MTX combined treatment can better control the condition of RA patients versus the control group, and it will not cause damage to the patient's blood system or liver/ kidney function. However, MTX alone or MTX combined treatment may increase the probability of adverse reactions in RA patients. There were some shortcomings in this study that should be noted. The number of included studies was small, and only involved a few indicators, which may limit the strength of our findings. Therefore, more large-scale multi- 
center clinical RCTs are needed in the future to determine the clinical efficacy of MTX in the treatment of RA.

\section{Acknowledgments}

Funding: The China Postdoctoral Science Foundation (2019 M663752); The Natural Science Foundation of Shannxi Province, China (2021JM-544).

\section{Footnote}

Reporting Checklist: The authors have completed the PRISMA reporting checklist. Available at https://dx.doi. org/10.21037/apm-21-2471

Conflicts of Interest: All authors have completed the ICMJE uniform disclosure form (available at https://dx.doi. org/10.21037/apm-21-2471). The authors have no conflicts of interest to declare.

Ethical Statement: The authors are accountable for all aspects of the work in ensuring that questions related to the accuracy or integrity of any part of the work are appropriately investigated and resolved.

Open Access Statement: This is an Open Access article distributed in accordance with the Creative Commons Attribution-NonCommercial-NoDerivs 4.0 International License (CC BY-NC-ND 4.0), which permits the noncommercial replication and distribution of the article with the strict proviso that no changes or edits are made and the original work is properly cited (including links to both the formal publication through the relevant DOI and the license). See: https://creativecommons.org/licenses/by-nc-nd/4.0/.

\section{References}

1. Kumar LD, Karthik R, Gayathri N, et al. Advancement in contemporary diagnostic and therapeutic approaches for rheumatoid arthritis. Biomed Pharmacother 2016;79:52-61.

2. Ferro F, Elefante E, Luciano N, et al. One year in review 2017: novelties in the treatment of rheumatoid arthritis. Clin Exp Rheumatol 2017;35:721-34.

3. Li C, Wang XR, Ji HJ, et al. Cardiovascular disease in rheumatoid arthritis: medications and risk factors in China. Clin Rheumatol 2017;36:1023-9.

4. Karami J, Aslani S, Jamshidi A, et al. Genetic implications in the pathogenesis of rheumatoid arthritis; an updated review. Gene 2019;702:8-16.

5. Kong XY, Wen CP. On Research Progress of Western and Chinese Medicine Treatment on Pre-Rheumatoid Arthritis. Chin J Integr Med 2019;25:643-7.

6. Pirmardvand Chegini S, Varshosaz J, Taymouri S. Recent approaches for targeted drug delivery in rheumatoid arthritis diagnosis and treatment. Artif Cells Nanomed Biotechnol 2018;46:502-14.

7. Nasonov EL. Prospects for rheumatoid arthritis pharmacotherapy: New opportunities and recommendations. Ter Arkh 2016;88:4-10.

8. Thakur S, Riyaz B, Patil A, et al. Novel drug delivery systems for NSAIDs in management of rheumatoid arthritis: An overview. Biomed Pharmacother 2018;106:1011-23.

9. Birkner B, Rech J, Stargardt T. Cost-utility analysis of deescalating biological disease-modifying anti-rheumatic drugs in patients with rheumatoid arthritis. PLoS One 2020;15:e226754.

10. Abbasi M, Mousavi MJ, Jamalzehi S, et al. Strategies toward rheumatoid arthritis therapy; the old and the new. J Cell Physiol 2019;234:10018-31.

11. Bastida C, Ruíz V, Pascal M, et al. Is there potential for therapeutic drug monitoring of biologic agents in rheumatoid arthritis? Br J Clin Pharmacol 2017;83:962-75.

12. Harigai M. Lymphoproliferative disorders in patients with rheumatoid arthritis in the era of widespread use of methotrexate: A review of the literature and current perspective. Mod Rheumatol 2018;28:1-8.

13. Alfaro-Lara R, Espinosa-Ortega HF, Arce-Salinas CA, et al. Systematic review and meta-analysis of the efficacy and safety of leflunomide and methotrexate in the treatment of rheumatoid arthritis. Reumatol Clin (Engl Ed) 2019;15:133-9.

14. Murosaki T, Nagatani K, Sato T, et al. Prediction of the therapeutic response to methotrexate at 24 weeks by methotrexate-polyglutamates concentration in erythrocytes at 8 weeks in patients with rheumatoid arthritis. Mod Rheumatol 2017;27:411-6.

15. Brady K, Qu Y, Stimson D, et al. Transition of Methotrexate Polyglutamate Drug Monitoring Assay from Venipuncture to Capillary Blood-Based Collection Method in Rheumatic Diseases. J Appl Lab Med 2019;4:40-9.

16. Rau R, Herborn G, Menninger H, et al. Progression in early erosive rheumatoid arthritis: 12 month results from a randomized controlled trial comparing methotrexate and gold sodium thiomalate. Br J Rheumatol 1998;37:1220-6.

17. Choi HK, Hernán MA, Seeger JD, et al. Methotrexate 
and mortality in patients with rheumatoid arthritis: a prospective study. Lancet 2002;359:1173-7.

18. Hoekstra M, van Ede AE, Haagsma CJ, et al. Factors associated with toxicity, final dose, and efficacy of methotrexate in patients with rheumatoid arthritis. Ann Rheum Dis 2003;62:423-6.

19. Aletaha D, Funovits J, Keystone EC, et al. Disease activity early in the course of treatment predicts response to therapy after one year in rheumatoid arthritis patients. Arthritis Rheum 2007;56:3226-35.

20. Kameda H, Ueki Y, Saito K, et al. Etanercept (ETN) with methotrexate (MTX) is better than ETN monotherapy in patients with active rheumatoid arthritis despite MTX therapy: a randomized trial. Mod Rheumatol 2010;20:531-8.

21. Bijlsma JWJ, Welsing PMJ, Woodworth TG, et al. Early rheumatoid arthritis treated with tocilizumab, methotrexate, or their combination (U-Act-Early): a multicentre, randomised, double-blind, double-dummy, strategy trial. Lancet 2016;388:343-55.

22. Burmester GR, Rigby WF, van Vollenhoven RF, et al. Tocilizumab in early progressive rheumatoid arthritis: FUNCTION, a randomised controlled trial. Ann Rheum Dis 2016;75:1081-91.

23. Xia Z, Lyu J, Hou N, et al. Iguratimod in combination with methotrexate in active rheumatoid arthritis : Therapeutic effects. Z Rheumatol 2016;75:828-33.

24. Giles JT. Extra-articular Manifestations and Comorbidity in Rheumatoid Arthritis: Potential Impact

Cite this article as: Jiang X, Zeng J, Chen F, Li J. Systematic review and meta-analysis on the efficacy of methotrexate in rheumatoid arthritis. Ann Palliat Med 2021;10(10):10652-10660. doi: 10.21037/apm-21-2471 of Pre-Rheumatoid Arthritis Prevention. Clin Ther 2019;41:1246-55.

25. Littlejohn EA, Monrad SU. Early Diagnosis and Treatment of Rheumatoid Arthritis. Prim Care 2018;45:237-55.

26. Zhao J, Li ZG. The challenges of early diagnosis and therapeutic prediction in rheumatoid arthritis. Int J Rheum Dis 2018;21:2059-62.

27. Wabe N, Wiese MD. Treating rheumatoid arthritis to target: physician and patient adherence issues in contemporary rheumatoid arthritis therapy. J Eval Clin Pract 2017;23:486-93.

28. De Cock D, Hyrich K. Malignancy and rheumatoid arthritis: Epidemiology, risk factors and management. Best Pract Res Clin Rheumatol 2018;32:869-86.

29. Chatzidionysiou K, Sfikakis PP. Low rates of remission with methotrexate monotherapy in rheumatoid arthritis: review of randomised controlled trials could point towards a paradigm shift. RMD Open 2019;5:e000993.

30. Aletaha D, Smolen JS. Diagnosis and Management of Rheumatoid Arthritis: A Review. JAMA 2018;320:1360-72.

31. Lortholary O, Fernandez-Ruiz M, Baddley JW, et al. Infectious complications of rheumatoid arthritis and psoriatic arthritis during targeted and biological therapies: a viewpoint in 2020. Ann Rheum Dis 2020;79:1532-43.

32. Vasconcelos LB, Silva MT, Galvao TF. Reduction of biologics in rheumatoid arthritis: a systematic review and meta-analysis. Rheumatol Int 2020;40:1949-59. 